\title{
Evaluation of Optical Flow Algorithms for Tracking Endocardial Surfaces on Three-Dimensional Ultrasound Data
}

\author{
Qi Duan ${ }^{\mathrm{a}}$, Elsa D. Angelini ${ }^{\mathrm{b}}$, Susan L. Herz ${ }^{\mathrm{a}}$, Christopher M. Ingrassia ${ }^{\mathrm{a}}$, Olivier Gerard ${ }^{\mathrm{c}}$, \\ Kevin D. Costa ${ }^{a}$, Jeffrey W. Holmes ${ }^{a}$, Andrew F. Laine ${ }^{a}$ \\ ${ }^{a}$ Department of Biomedical Engineering, Columbia University, ET351, 1210 Amsterdam Avenue, \\ New York, NY, USA, 10027; \\ ${ }^{b}$ Ecole Nationale Supérieure des Télécommunications, Département Traitement du Signal et des \\ Images (TSI), 46 rue Barrault, Paris, France, 75013; \\ ${ }^{c}$ Philips France, 51 rue Carnot, BP 301, Suresnes, France, 92156
}

\begin{abstract}
With relatively high frame rates and the ability to acquire volume data sets with a stationary transducer, 3D ultrasound systems, based on matrix phased array transducers, provide valuable three-dimensional information, from which quantitative measures of cardiac function can be extracted. Such analyses require segmentation and visual tracking of the left ventricular endocardial border. Due to the large size of the volumetric data sets, manual tracing of the endocardial border is tedious and impractical for clinical applications. Therefore the development of automatic methods for tracking three-dimensional endocardial motion is essential. In this study, we evaluate a four-dimensional optical flow motion tracking algorithm to determine its capability to follow the endocardial border in three dimensional ultrasound data through time. The four-dimensional optical flow method was implemented using three-dimensional correlation. We tested the algorithm on an experimental open-chest dog data set and a clinical data set acquired with a Philips' iE33 three-dimensional ultrasound machine. Initialized with left ventricular endocardial data points obtained from manual tracing at end-diastole, the algorithm automatically tracked these points frame by frame through the whole cardiac cycle. A finite element surface was fitted through the data points obtained by both optical flow tracking and manual tracing by an experienced observer for quantitative comparison of the results. Parameterization of the finite element surfaces was performed and maps displaying relative differences between the manual and semi-automatic methods were compared. The results showed good consistency between manual tracing and optical flow estimation on $73 \%$ of the entire surface with fewer than $10 \%$ difference. In addition, the optical flow motion tracking algorithm greatly reduced processing time (about $94 \%$ reduction compared to human involvement per cardiac cycle) for analyzing cardiac function in threedimensional ultrasound data sets.
\end{abstract}

Keywords: Real-time three-dimensional ultrasound, optical flow, finite element model, matrix phased array

\section{INTRODUCTION}

Among all the modalities in clinical cardiac screening, ultrasound has the highest time resolution. However, most clinical examinations are still limited to the analysis of 2D ultrasound images. Developments in 3D echocardiography started in the late 1980s with the introduction of off-line 3D medical ultrasound imaging systems. Many review articles have been published over the past decade, assessing the progress and limitations of 3D ultrasound technology for clinical screening ${ }^{1-4}$. These articles reflect the diversity of 3D systems that were developed for both image acquisition and reconstruction. Although 2D transducers can be configured to assemble a 3D volume from a series of planar views, only matrix phased array transducers can scan a true three-dimensional volume at each pulse ${ }^{5}$. This technology was fundamentally different from the former generations of 3D systems as the entire cardiac volume was acquired in real-time ( 1 scan for 1 cardiac cycle), enabling the cardiologist to view moving cardiac structures from any given plane in real-time. A first generation of real-time three-dimensional ultrasound (RT3D) scanners was introduced in early 1990s by Volumetrics ${ }^{5}$ but acquisitions artifacts prevented the technology from meeting its initial expectation and reaching its full potential. A new generation of RT3D transducers was introduced by Philips Medical Systems (Best, The Netherlands) in 2000 with the SONOS 7500 transducer that can acquire a fully sampled cardiac volume in four cardiac cycles. Each scan produces $1 / 4$ of the cardiac volume so that 4 scans are performed and spatially aggregated to generate one ultrasound volume over one 
cardiac cycle. This technical design enabled to dramatically increase spatial resolution and image quality. With this new technology, real-time three-dimensional ultrasound based on matrix phased array transducers is likely to become a predominant technique for dynamic patient screening in cardiac and obstetric practice within the next few years. With a fairly high frame rate and the ability to acquire volume data using a single stationary probe, real-time three-dimensional ultrasound provides unique and valuable information, which is ideally suited to quantitatively extract the complex threedimensional motion of the heart. Abnormal ventricular wall motion can be detected via quantitative four-dimensional analysis of the endocardial surface and computation of local fractional shortening ${ }^{6}$. Such quantitative analysis requires tracking and segmentation of the endocardial surfaces. However, manual tracing of endocardial borders is tedious and impractical in clinical applications, while reliable automated segmentation methods are not available yet. In this context we propose to apply a motion tracking algorithm ${ }^{7}$, based on optical flow, as an alternative to manual segmentation of the endocardial surface to efficiently and accurately extract endocardial motion information from the large volumetric data sets obtained from 3D ultrasound. We tested the four-dimensional optical flow algorithm on an open-chest dog data with infarct and a clinical echocardiography data. We compared endocardial surfaces manually segmented by an expert operator with those predicted by the automated tracking algorithm, and report differences in terms of qualitative visual discrepancies and quantitative relative differences in reconstructed endocardial surfaces derived from finite element fitting of segmented surface data.

\section{METHOD}

\subsection{Pre-processing}

The presence of speckle noise patterns makes the interpretation of ultrasound images, either by a human operator or with a computer-based system, very difficult. It is highly desirable for certain applications such as automatic motion tracking, to apply some denoising prior to scan conversion in order to remove speckle noise artifacts and improve signal homogeneity within distinct anatomical tissues. Our group has presented previous work on applying a computationally efficient denoising filter based on anisotropic diffusion ${ }^{8}$, a method which is very efficient for speckle reduction in ultrasound and radar images.

Anisotropic diffusion methods apply the following heat-diffusion type of dynamic equation to the gray levels of a given 3D image data $I(x, y, z, t)$ :

$$
\frac{\partial I}{\partial t}=\operatorname{div}(c(x, y, z, t) \nabla I)
$$

where $c(x, y, z, t)$ is the diffusion parameter, div denotes the divergence operator, and $\nabla I$ denotes the gradient of the image intensity. In the original work of Perona and Malik ${ }^{9,10}$, the concept of anisotropic diffusion was introduced with the selection of a variable diffusion parameter, function of the gradient of the data:

$$
c(x, y, z, t)=g(|\nabla I(x, y, z, t)|)
$$

We used the diffusion function proposed by Weickert ${ }^{11}$ defined as:

$$
g(x, \lambda)=\left\{\begin{array}{ll}
1 & x \leq 0 \\
1-e^{-\frac{3.315}{(x / \lambda)^{4}}} & x>0
\end{array} .\right.
$$

The parameter $\lambda$ serves as a gradient threshold, defining edge points $x_{k}$ as locations where $\left|\nabla I_{x_{k}}\right|>\lambda$. This bellshaped diffusion function acts as an edge-enhancing filter, with high diffusion values in smooth areas and low values at edge points. A linear model for the threshold was used:

$$
\lambda(t)=\lambda_{0}+a t,
$$

with $\lambda_{0}$ an initial gradient value, $a$ is a slope parameter and $t$ is the time iteration index. Parameters were set empirically for the data sets processed as $\lambda_{0}=5, a=10$, and 10 iterations were performed for each $3 \mathrm{D}$ frame.

\subsection{Correlation-based Optical Flow}


Optical flow (OF) tracking refers to the computation of the displacement field of objects in an image, based on the assumption that the intensity of the object remains constant. In this context, motion of the object is characterized by a flow of pixels with constant intensity. The assumption of intensity conservation is typically unrealistic for natural movies and medical imaging applications, motivating the argumentation that optical flow can only provide qualitative estimation of object motions. There are two global families of optical flow computation techniques: (1) Differential techniques ${ }^{12-14}$ that compute velocity from spatio-temporal derivatives of pixel intensities; (2) Region-based matching techniques ${ }^{15,16}$, which compute the optical flow via identification of local displacements that provide optimal correlation of two consecutive image frames. Compared to differential optical flow approaches, region-based methods using correlation measures are less sensitive to noisy conditions and fast motion ${ }^{7}$ but assume that displacements in small neighborhood are similar. In the context of three-dimensional ultrasound, this later approach appeared more appropriate and was selected for this study. Given two data sets from consecutive time frames: $(I(\mathbf{x}, t), I(\mathbf{x}, t+\Delta t))$, the displacement vector $\Delta \mathbf{x}$ for each pixel in a small neighborhood $\Omega$ around a pixel $x$ is estimated via maximization of the cross-correlation coefficient defined as:

$$
r=\frac{\sum_{\mathbf{x} \in \Omega}\left(I(\mathbf{x}, t)^{*} I(\mathbf{x}+\Delta \mathbf{x}, t+\Delta t)\right)}{\sqrt{\sum_{\mathbf{x} \in \Omega} I^{2}(\mathbf{x}, t) \sum_{\mathbf{x} \in \Omega} I^{2}(\mathbf{x}+\Delta \mathbf{x}, t+\Delta t)}}
$$

In this study, correlation-based OF was applied to estimate the displacement of selected voxels between two consecutive ultrasound volumes in the cardiac cycle. The search window $\Omega$ was centered at every $(5 \times 5 \times 5)$ pixel volume and was set to size $(7 \times 7 \times 7)$. The optical flow algorithm is four-dimensional in the sense of that it utilizes $3 \mathrm{D}$ plus time data.

\subsection{Segmentation}

An expert performed manual tracing of the endocardial surface, for all time frames, on rotating B-scan views (long-axis views rotating around the central axis of the ventricle) and C-scan views (short-axis views at different depths).

\subsection{Tracking with optical flow}

The proposed optical flow method was initialized using the manually traced surfaces at end-diastole (ED). Starting with a set of endocardial surface points (about 3000 points, roughly $1 \mathrm{~mm}$ apart for manual tracing) defined at ED; the optical flow algorithm was used to track the surface in time through the whole cardiac cycle.

\subsection{Surface reconstruction based on finite element model}

To visualize and quantify the difference between endocardial surfaces tracked with manual segmentation and with optical flow, we parameterized the endocardial surface in prolate spheroidal coordinates ${ }^{17}$. The endocardial surfaces were registered using three manually selected anatomical landmarks: the center of the mitral orifice, the endocardial apex, and the equatorial mid-septum. Each surface was then fit to a finite element model (FEM) in prolate spheroidal coordinates $(\lambda, \mu, \theta)$, projecting the radial coordinate $\lambda$ to a 64 -element surface mesh with bicubic Hermite interpolation to yield a realistic 3D endocardial surface using the custom finite element package Continuity 5.5 developed at the University of California San Diego (http://cmrg.ucsd.edu). For each time frame, root mean squared errors (RMSE) of difference of the radial coordinate $\lambda$ at node points, for OF and manual tracing results were computed. Ventricular volumes were also computed from the manually traced and the tracked endocardial surfaces. Finally the radial nodal values and first derivatives were used to map the normalized difference between the two surfaces, $\varepsilon=\left(\lambda_{\text {manual }}-\lambda_{\text {optical }}\right) / \lambda_{\text {manual }}$ using custom Matlab software. This mapping, displayed as a Hammer map, preserves relative areas of the flattened endocardial surface ${ }^{6}$.

\section{DATA}

The proposed tracking approach was tested on two data sets acquired with an iE33 3D ultrasound machine (Philips Medical Systems, Best, the Netherlands):

- One data set on an anesthetized open-chest dog acquired 2 minutes after induction of ischemia via occlusion of the proximal left anterior descending coronary artery. This data set was acquired by positioning the transducer 
directly on the apex of the heart, providing high image quality and a small field of view. Spatial resolution of the analyzed data was $\left(0.56 \mathrm{~mm}^{3}\right)$ and 16 frames were acquired for one cardiac cycle.

- One transthoracic clinical data set from a heart-transplant patient. Spatial resolution of the analyzed data was $\left(0.8 \mathrm{~mm}^{3}\right)$ and 16 frames were acquired for one cardiac cycle.

Data acquisition was triggered with the EKG initialized at ED (Frame 1).

Because of the smaller field of view used to acquire the open-chest dog data and the positioning of the transducer directly on the dog's heart, image quality was significantly higher in this data set, with some fine anatomical structures visible. Cross-section views at end-diastole (ED) from the open-chest baseline data set, and the patient data set are shown in Figure 1.

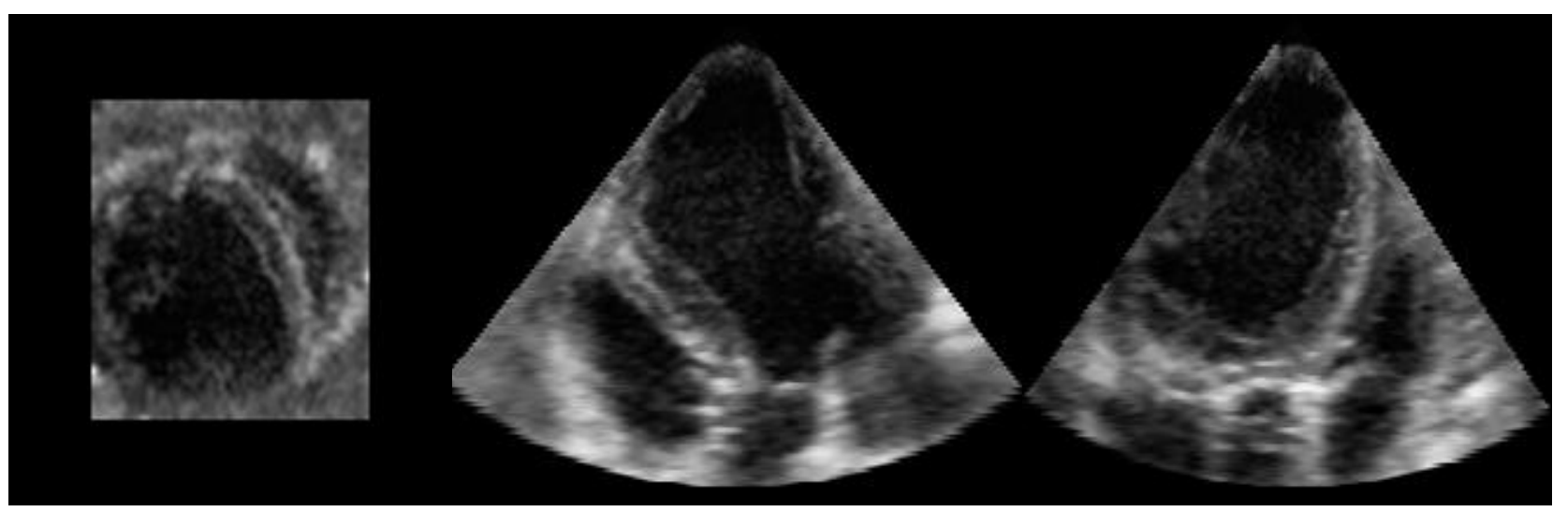

(a)

(b)

(c)

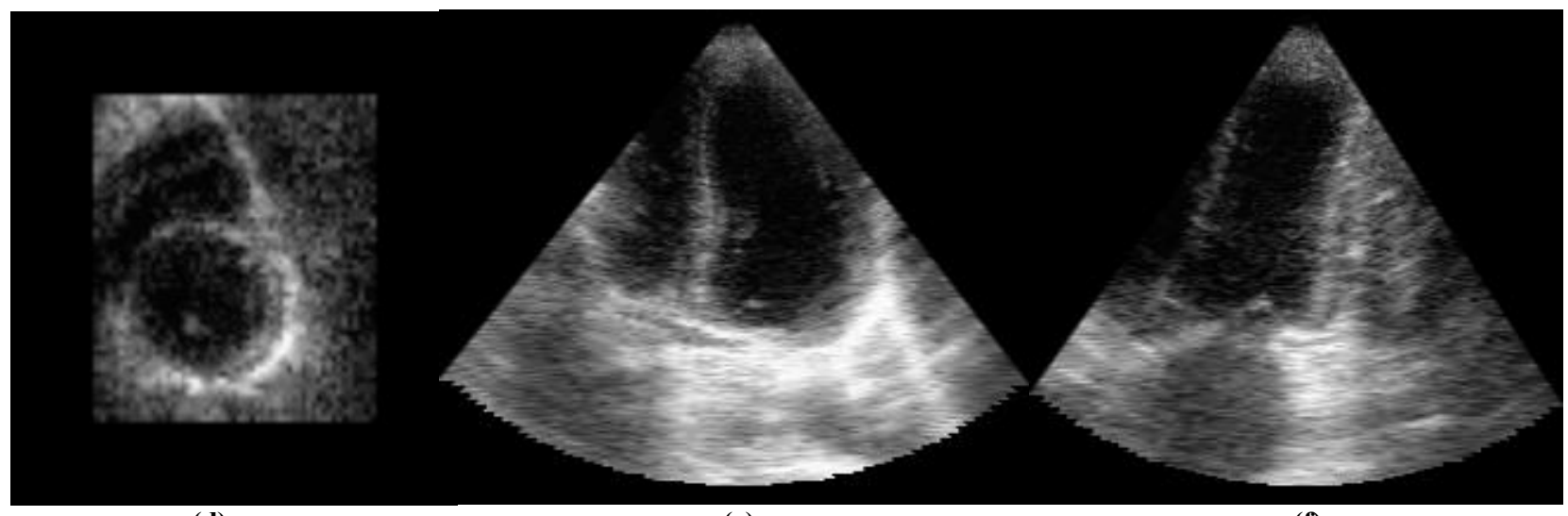

(d)

(e)

(f)

Figure 1. Cross-section views at ED for (a-c) Open-chest dog data, after ischemia, (d-f) Patient with transplanted heart. (a, d) axial, (b, e) elevation and (c, f) azimuth views.

\section{RESULTS}

\subsection{Open-chest dog data}

RMSE results reported a maximum difference on radial absolute difference of 0.08 (average radial coordinate value was $0.7 \pm 0.3$ at ED and $0.6 \pm 0.2$ at ES) on the dog post-ischemia data set. Maximum LV volume differences were less than $5 \mathrm{ml}$. RMSE values were smaller for OF tracking on larger volumes. Figure 2 shows the endocardial surface generated from optical flow tracking (dark gray) and manual tracing (light gray) at ES (frame 07) in two different views. The two methods yield very close agreement through most of the endocardial surface, although the optical flow produced slightly smaller volumes than manually tracing. As a result, the optical flow algorithm underestimated ES volume by about $11.05 \%$, and the ejection fraction (EF) values were $34.3 \%$ for manual tracing and $41.6 \%$ for optical flow tracking. 
We present, in a series of figures below, results of comparison of the endocardial geometry of the surfaces extracted with the two methods. In Figure 2, we plotted RMSE and LV volumes through the whole cardiac cycle, showing very high agreement of the two methods.

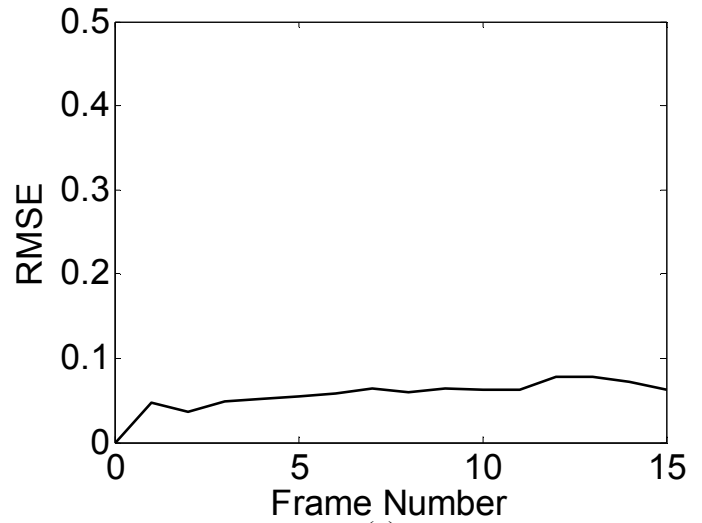

(a)

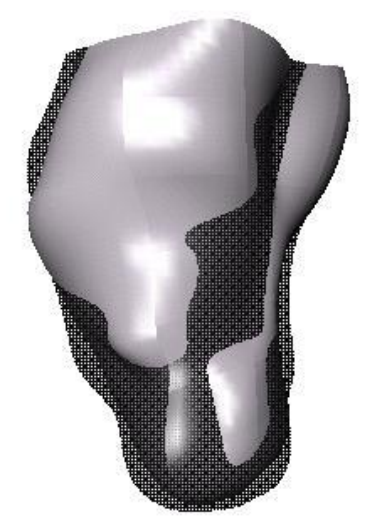

(c)

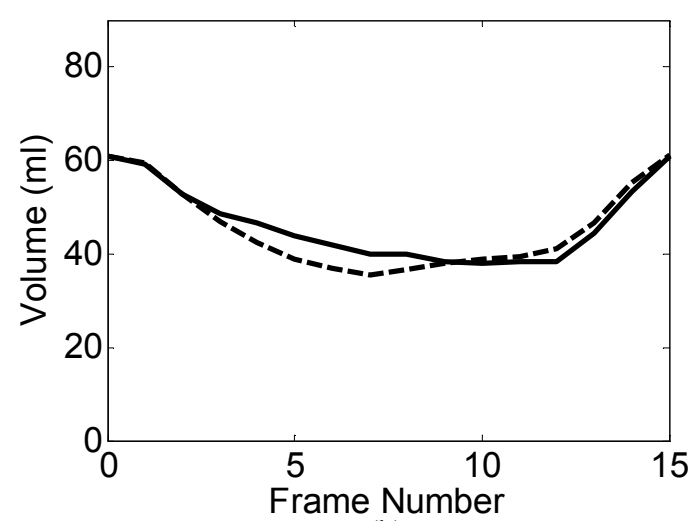

(b)

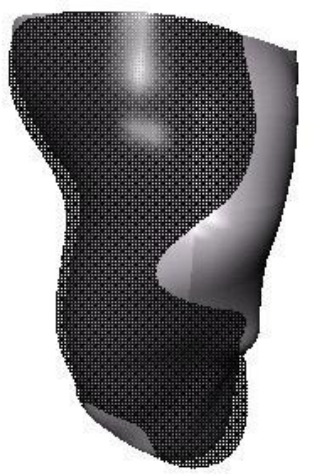

(d)

Figure 2. Results on post-ischemia open-chest dog data sets. (a) RMSE of radial absolute differences; (b) LV volumes from manual tracing (solid line), and OF tracking (dashed line); Three-dimensional rendering of endocardial surfaces at ES were generated from manual tracing (dark gray) and OF tracking (light gray) for (c) lateral views and (d) anterior views.

In Figure 3, we present a Hammer map of the radial relative difference. This more quantitative and detailed surface comparison reveals that most parts of the surface differ by less than $20 \%$ except near the base. Overall $39 \%$ of endocardial surfaces differ by less than $5 \%$ between the two methods and $72 \%$ of the entire surface differs by less than $10 \%$. 


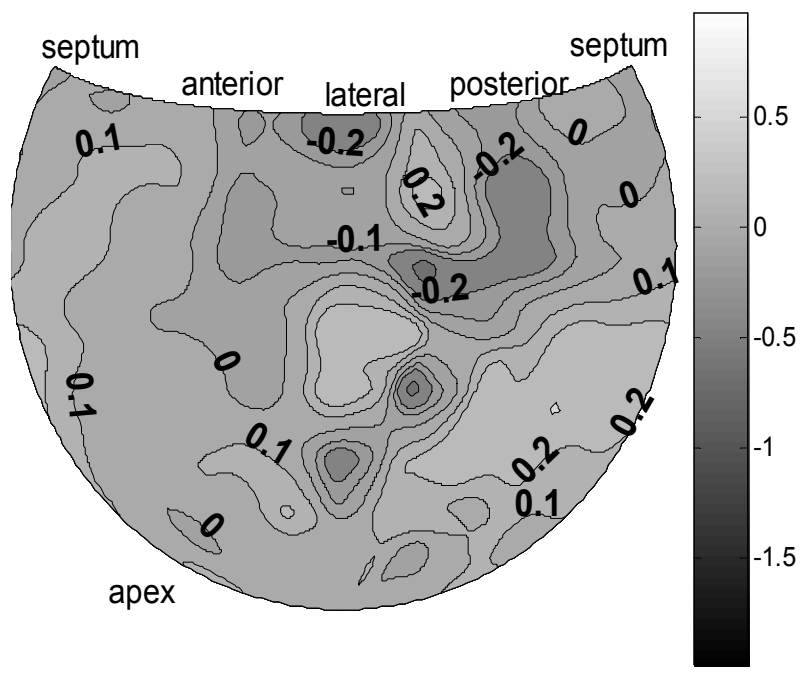

Figure 3. Relative difference maps between OF and manual tracing surfaces at ES on post-ischemia open-chest dog data sets.

Motion amplitude maps for all frames were also generated using a similar fitting technique. The result is shown in Figure 4. The motion amplitude is referring to the magnitude of motion vector for each pixel between two consecutive frames. From the motion maps in Figure 4, we could roughly identify the different phase of the cardiac cycle: rapid ejection during frame 00-03 (a-d); reduced ejection during frame 04-05 (e-f); end-systole at frame 06 (g); isovolumetric ventricular relaxation during frame 07-08 (h-i); rapid filling during frame 09-13 (j-n); and reduced filling beginning at frame 14 (o). Also from the motion maps, we could guess that there was some abnormal motion at part of the anterior wall and part of the posterior wall near the apex. This conclusion matches the fact that there was occlusion of the proximal left anterior descending coronary artery. 


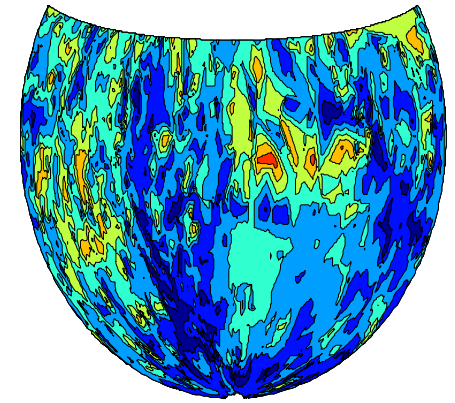

(a)
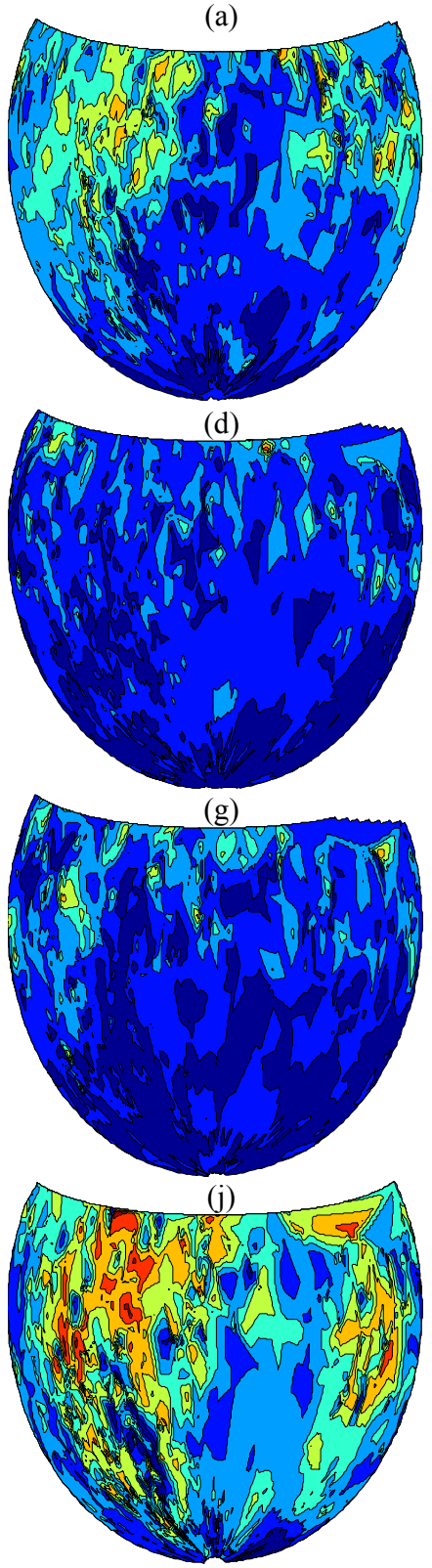

(m)
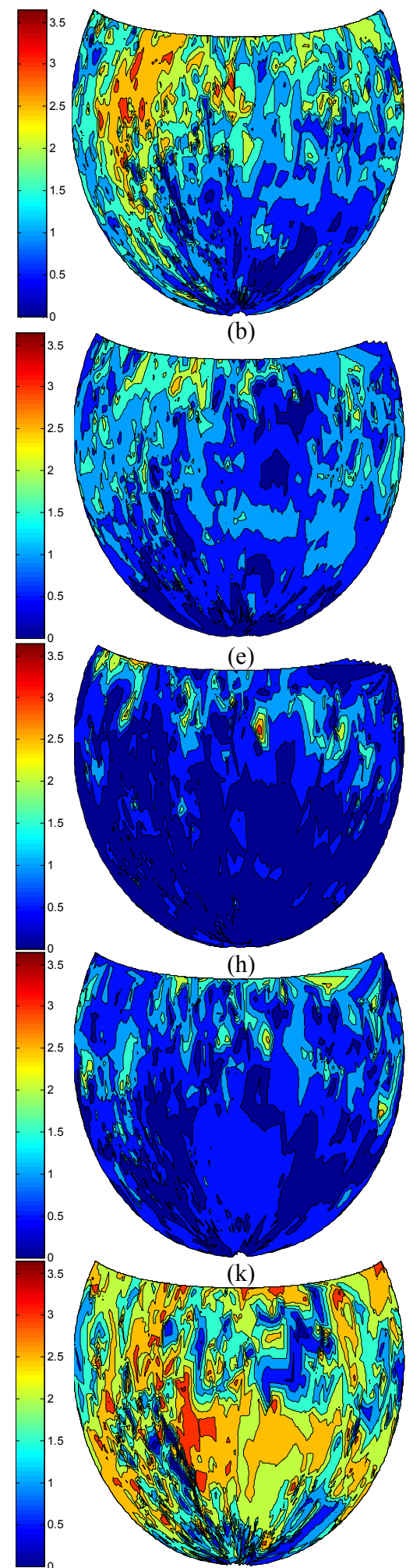

(n)

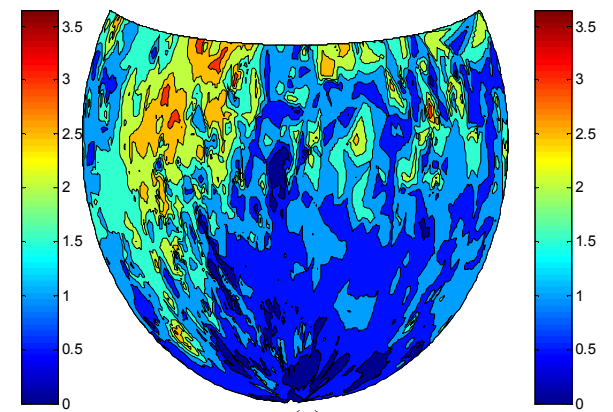

(c)
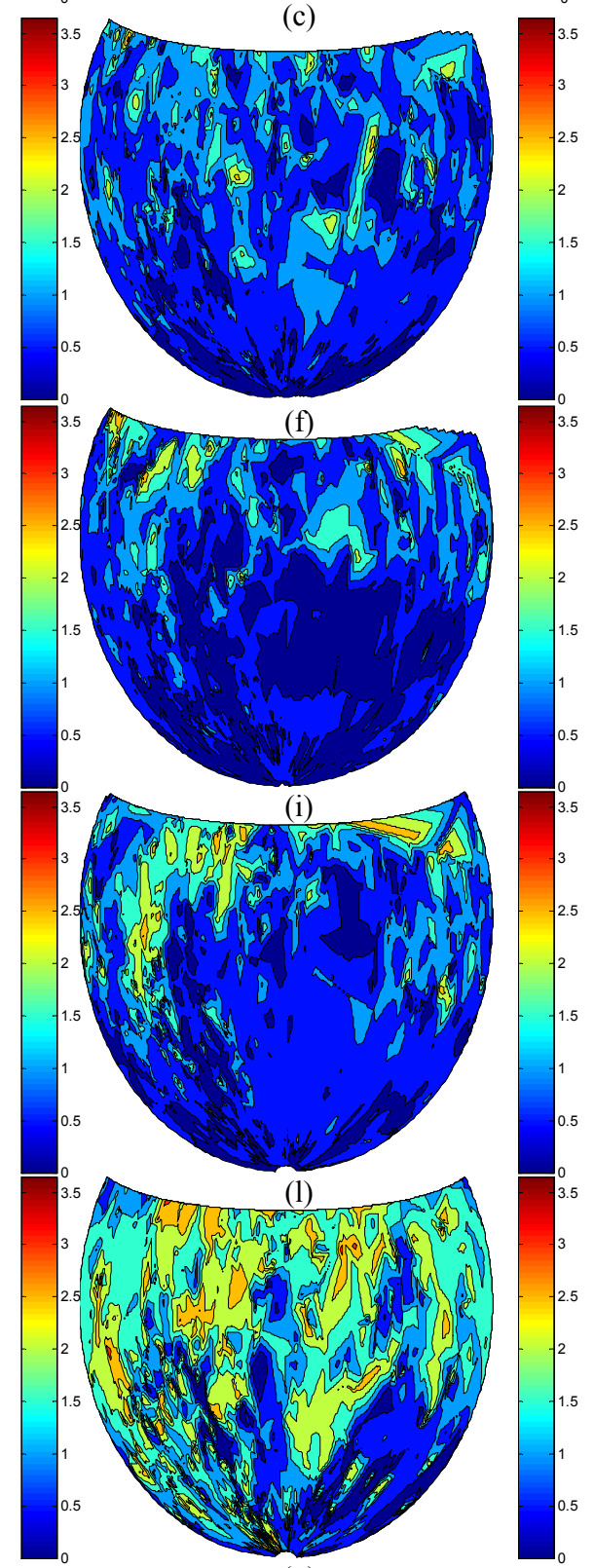

(o)

Figure 4. Motion amplitude maps, derived from OF tracking, for each frame on the post-ischemia open-chest dog data. (a)-(o): frame00-15. 


\subsection{Clinical data}

RMSE results reported a maximum difference on radial difference of 0.09 (average radial coordinate value was $0.6 \pm 0.2$ at ED and $0.5 \pm 0.2$ at ES) at frame 09 (after end-systole) on the clinical data set. Maximum LV volume differences was less than $10 \mathrm{ml}$. Figure 5 shows the endocardial surface generated from optical flow tracking (dark gray) and manual tracing (light gray) at ES (frame 06) in two different views. The two methods yield very close agreement through most of the endocardial surface, and the OF generated larger volumes, overestimating ES volumes by about $2.51 \%$. Calculated EF were $46.00 \%$ for manual tracing and $44.65 \%$ for optical flow tracking.

A much more quantitative and detailed surface comparison is provided on the Hammer maps of radial differences, shown in Figure 6. From these maps, we can clearly see that most parts of the surface differ by less than $20 \%$ except near the apex, where the endocardial border approaches the limits of the field of view. Overall $47 \%$ of endocardial surfaces differ by less than $5 \%$ between the two methods and $73 \%$ of the entire surface differs by less than $10 \%$.

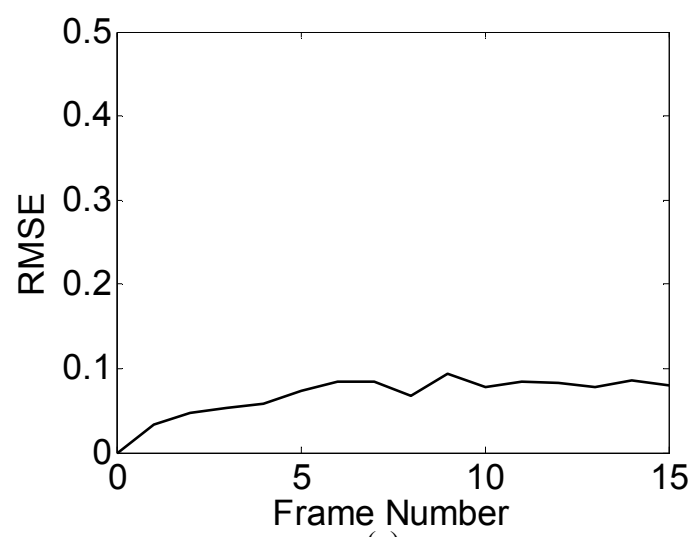

(a)

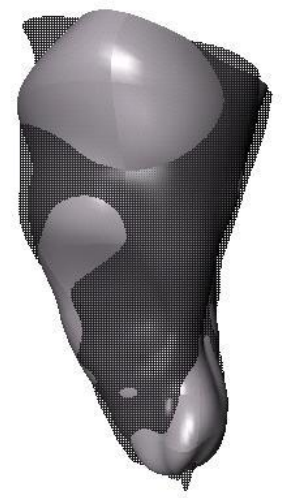

(c)

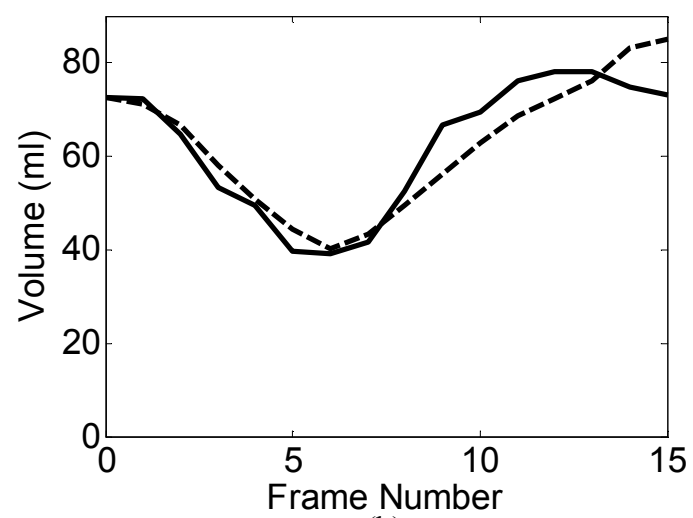

(b)

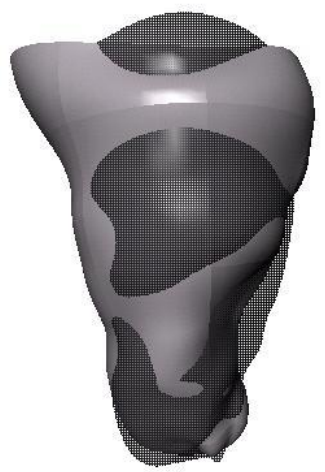

(d)

Figure 5. Results on the clinical data set. (a) RMSE of radial absolute differences; (b) LV volumes from manual tracing (solid line), and OF tracking (dashed line); Three-dimensional rendering of endocardial surfaces at ES were generated from manual tracing (dark gray) and OF tracking (light gray) for (c) lateral views and (d) anterior views 


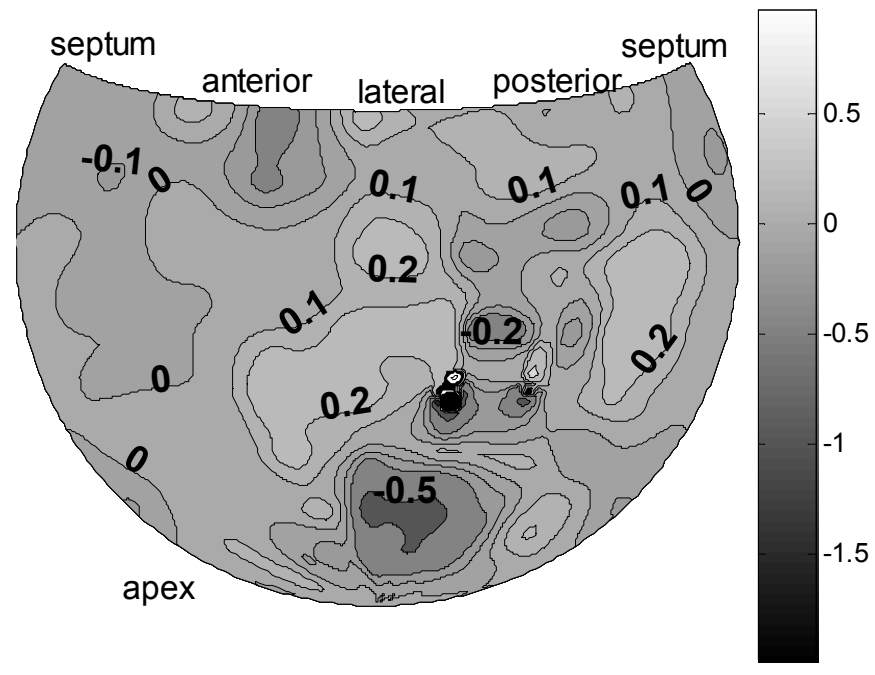

Figure 6. Relative difference maps between OF and manual tracing surfaces at ES on clinical patient data.

We also tried to track the epicardium on this clinical data set. However, due to the limited field of view, only a small portion of the epicardial surface was visible. An operator traced the visible part of the epicardial surface on all frames. The OF was initialized on the first frame (ED) and tracked the given initial surface automatically over the cardiac cycle. The epicardial surfaces generated by the two methods were compared at ES, via again modelization onto a 16 FEM expressed in prolate spheroidal coordinates. Results are shown in Figure 7, revealing good visual agreement between the two surfaces.

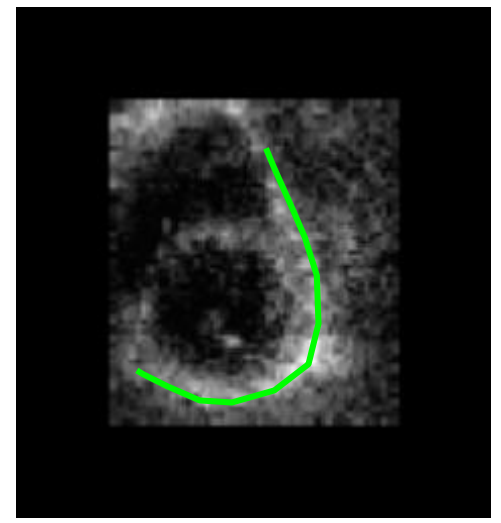

(a)

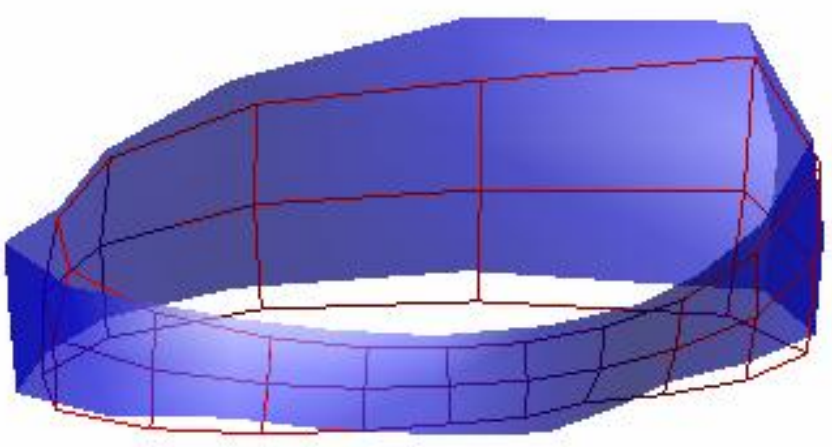

(b)

Figure 7. (a) Illustration of manually traced epicardium surface; (b) 3D visualization of epicardial surfaces reconstructed from manual tracing (blue continue surface) and OF tracking (red wire-frame) at ES frame by cubic spline interpolation. 


\subsection{Computational Time}

Computational time required for the optical flow algorithm was about 1 minute per frame, whereas the processing time required for manual tracing was about 10 minutes per frame. The total processing time needed was:

- $\quad$ For the human expert

- 70 minutes for all seven frames during the ejection phase.

- 160 minutes for all sixteen frames during the whole cardiac cycle

- $\quad$ For the Optical Flow tracking

○ 16 minutes for the ejection phase

- 25 minutes for the whole cardiac cycle (including 10 minutes needed for manual initialization for the first frame).

All computations were implemented in "C" and executed on a 2.4GHz 64-bit AMD server, running Red Hat Linux Enterprise AS. This comparison clearly demonstrates the need for automated or semi-automated segmentation and tracking tool for myorcardial surfaces, Optical flow appears as a very strong candidate with reasonable computational times. We expect great improvements in terms of computation speed when implementing the method in a multiresolution framework.

\section{CONCLUSION}

A four-dimensional optical flow algorithm was tested using three-dimensional ultrasound data sets for tracking the myocardium on an open-chest dog and a clinical exam. Qualitative and quantitative evaluations of the tracking algorithm were performed and showed consistency between the manually traced endocardial surfaces and the surface obtained through tracking of the ED surface over the cardiac cycle. The proposed four-dimensional optical flow algorithm could successfully track the left ventricular endocardial surface from three-dimensional volumetric ultrasound data. This capability takes full advantage of the comprehensive three-dimensional motion information embedded in real-time volumetric ultrasound data for tracking cardiac wall motion. Furthermore, as a semi-automatic method, the algorithm can substantially reduce processing time needed from cardiologists to extract quantitative information from the data (as much as $85 \%$ time-saving for the ejection phase and $94 \%$ for the whole cardiac cycle). Motion amplitude maps generated from OF estimation provided a vivid visualization of the distribution of ventricular motion over time. A novel approach for comparing two endocardial surfaces using finite element reconstruction was also proposed. Using finite element reconstruction and radial difference Hammer maps, quantitative comparison of two segmented surfaces can be achieved and regional differences readily visualized. As a semi-automatic method, the combination of finite element representation and four-dimensional optical flow tracking will pave the path for clinical application of real-time threedimensional ultrasound in quantitative cardiac motion analysis.

\section{ACKNOWLEDGEMENT}

This work was funded by National Science Foundation grant BES-02-01617, American Heart Association \#0151250T, Philips Medical Systems, New York State NYSTAR/CAT Technology Program, and the Louis Morin Fellowship program. Dr. Andrew McCulloch at the University of California San Diego provided the finite element software "Continuity" through the National Biomedical Computation Resource (NIH P41RR08605). The authors also would like to thank Drs. Shunichi Homma and Todd Pulerwitz (Department of Medicine, Columbia University).

\section{REFERENCE}

1. E. O. Ofili and N. C. Nanda, "Three-dimensional and four-dimensional echocardiography," Ultrasound Medical Biology, vol. 20, 1994.

2. A. Fenster and D. B. Downey, "Three-Dimensional Ultrasound Imaging," in Handbook of Medical Imaging. Volume1. Physics and Psychophysics, vol. 1, H. L. K. Jacob Beutel, Richard L. Metter, Ed. Bellingham, WA, USA.: SPIE- The International Society of Optical Engineering, 2000, pp. 463-510.

3. R. N. Rankin, A. Fenster, D. B. Downey, P. L. Munk, M. F. Levin, and A. D. Vellet, "Three-dimensional sonographic reconstruction: technique and diagnostic applications," American Journal of Radiology, vol. 161, pp. 695-702, 1993. 
4. M. Belohlavek, D. A. Foley, T. C. Gerber, T. M. Kinter, J. F. Greenleaf, and J. B. Seward, "Three- and fourdimensional cardiovascular ultrasound imaging: a new era for echocardiography," Mayo Clinic Proceedings, vol. 68, pp. 221-240, 1993.

5. O. T. V. Ramm and S. W. Smith, "Real time volumetric ultrasound imaging system," Journal of Digital Imaging, vol. 3, pp. 261-266, 1990.

6. S. Herz, T. Pulerwitz, K. Hirata, A. Laine, M. DiTullio, S. Homma, and J. Holmes, "Novel Technique for Quantitative Wall Motion Analysis Using Real-Time Three-Dimensional Echocardiography," in Proceedings of the 15th Annual Scientific Sessions of the American Society of Echocardiography, 2004.

7. J. L. Barron, D. Fleet, and S. Beauchemin, "Performance of optical flow techniques," Int. Journal of Computer Vision, vol. 12, pp. 43-77, 1994.

8. Q. Duan, E. D. Angelini, and A. Laine, "Assessment of visual quality and spatial accuracy of fast anisotropic diffusion and scan conversion algorithms for real-time three-dimensional spherical ultrasound," in SPIE International Symposium Medical Imaging, 5373, 331-342, San Diego, CA, USA, 2004.

9. P. Perona and J. Malik, "Scale space and edge detection using anisotropic diffusion," in IEEE Workshop on Computer Vision, 1, 16-22, 1987.

10. P. Perona and J. Malik, "Scale-space and edge detection using anisotropic diffusion," IEEE Transactions on Pattern Analysis and Machine Intelligence, vol. 12, pp. 629-639, 1990.

11. J. Weickert, B. M. t. H. Romeny, and M. A. Viergever, "Efficient and reliable schemes for nonlinear diffusion filtering," IEEE Transactions on Image Processing, vol. 7, pp. 398-410, 1998.

12. B. D. Lucas and T. Kanade, "An iterative image registration technique with an application to stereo vision," in International Joint Conference on Artificial Intelligence (IJCAI), 674-679, 1981.

13. B. K. P. Horn and B. G. Schunck, "Determining optical flow," Artificial Intelligence, vol. 17, 1981.

14. H. Nagel, "Displacement vectors derived from second-order intensity variations in image sequences," Computer Vision Graphics Image Processing, vol. 21, pp. 85-117, 1983.

15. P. Anandan, "A computational framework and an algorithm for the measurement of

16. $\quad$ visual motion," International Journal of Computer Vision, vol. 2, pp. 283-310, 1989.

17. A. Singh, "An estimation-theoretic framework for image-flow computation," in International Conference on Computer Vision, 168-177, 1990.

18. C. M. Ingrassia, S. L. Herz, K. D. Costa, and J. W. Holmes, "Impact of Ischemic Region Size on Regional Wall Motion," in Proceedings of the 2003 Annual Fall Meeting of the Biomedical Engineering Society, 2003. 